\title{
Resource Allocation Optimization of UAVs-Enabled Air-Ground Collaborative Emergency Network in Disaster Area
}

\author{
Lei $\mathrm{Wu}^{\mathrm{a}}$ and Weijian Wang \\ ${ }^{a}$ School of Information Management, Beijing Information Science and Technology University, Beijing, 100192, China \\ ${ }^{b}$ College of Information and Communication Engineering, Harbin Engineering University, Harbin, 150000, China
}

\begin{abstract}
This paper aims to improve the efficiency of UAVs-enabled air-ground collaborative emergency networks through resource allocation optimization. The bandwidth assignment, UAVs' flight trajectories, and transmission power were jointly optimized to maximize the achievable signal rate of all terminal receivers in the downlink. The block coordinate descent method and successive convex approximation were utilized to solve the established mixed-integer non-convex optimization problem. Then, an emergency communication scenario was designed to run different emulation experiments, which were used to verify that the proposed algorithm was effective. Numerous results showed that the proposed algorithm can attain great performances in this scenario. Compared with single factor optimizing strategies, the users' signal rate can be improved by 5\%-28\% through joint optimization.
\end{abstract}

Keywords: UAV; air-ground collaborative network; resource allocation; signal rate maximization; non-convex problem; mix-integer optimization

(Submitted on June 11, 2019; Revised on July 15, 2019; Accepted on August 10, 2019)

(C) 2019 Totem Publisher, Inc. All rights reserved.

\section{Introduction}

Unmanned aerial vehicles (UAVs) have undergone rapid development and have attracted wide attention for various applications, such as cargo delivery, aerial photography, agricultural irrigation, and wire monitoring. Characterized by flexible deployment and low cost, UAVs can greatly advance the development of wireless communication [1-2]. In particular, UAVs have high potential for creating line-of-sight (LoS) communication links, which can achieve better channel quality. Meanwhile, massive research efforts from academia have been devoted to the emerging collaborative airground collaborative networks, which can benefit from the high stability of terrestrial networks as well as the flexible mobility and LoS links of UAVs.

UAVs normally serve as aerial base stations or aerial relays [3-4]. In [5], Al-Hourani proposed an analytical approach to maximize wireless network coverage by optimizing the altitude of a single UAV. In [6], Mozaffari et al. extended the study to the altitude optimization of multiple UAVs. The authors in [7] provided a method to find optimal locations for UAVs in a 3D space. [8] and [9] focused on the trajectory optimization of single UAV relay system. As for multi-user situations, the authors in [10] proposed a cyclical multiple access scheme to realize the association and schedule of multiple users. In [11], Xue et al. created an IoT scenario for UAV data transmission and considered the probability of creating LoS links, which enriched the system model. In [12], Wu et al. studied the multi-UAVs trajectory optimization and power control to avoid severe interference. Finally, in [13], Xue turned to the cooperation of aerial platforms and terrestrial stations. However, the research only involved a single UAV's route planning.

Previous studies have not dealt with multiuser bandwidth assignment jointly with multiple UAVs' mobility and energy budget in air-ground collaborative networks, which are critically important for the cooperation between terrestrial base stations and UAVs. Driven by this concern, we focus on the resource allocation optimization of UAVs-enabled air-ground collaborative emergency networks in this paper. Aiming at the maximization of signal rate of all users, the block coordinate

\footnotetext{
* Corresponding author.

E-mail address: 77476456@qq.com
} 
descent method and successive convex approximation are employed to solve the complex optimization problem by transforming it into three convex problems.

\section{Model and Problem Formulation}

\subsection{Scenario Description}

As depicted in Figure 1, we consider the following emergency communication scenario: in a square area centered by a ground base station, several terminal receivers are distributed and need to communicate externally. In order to simplify the problem, terminal receivers (TRs) are seen as stationary. Due to earthquakes, debris flow, tornados, or other disasters, the ground base station (GBS) may be partially damaged, and the bearing capacity may be weakened. In addition, disasters may lead to serious blockage between terminal receivers and ground base stations and result in poor channel quality. In order to meet the communication demand of disaster areas, a number of drones can be sent out to serve as aerial mobile base stations for assistance. These aerial base stations can fly along a certain trajectory in the area, which enables them to get as close as possible to TRs to obtain higher channel quality with line of sight (LoS) links. Our task is to optimize the bandwidth assignment of the base stations and the UAVs' trajectory and transmission power to improve the communication capacity of the entire network. Excluding the cases that need to be distinguished, the aerial base station and the ground base station are collectively referred to as the base station hereinafter.

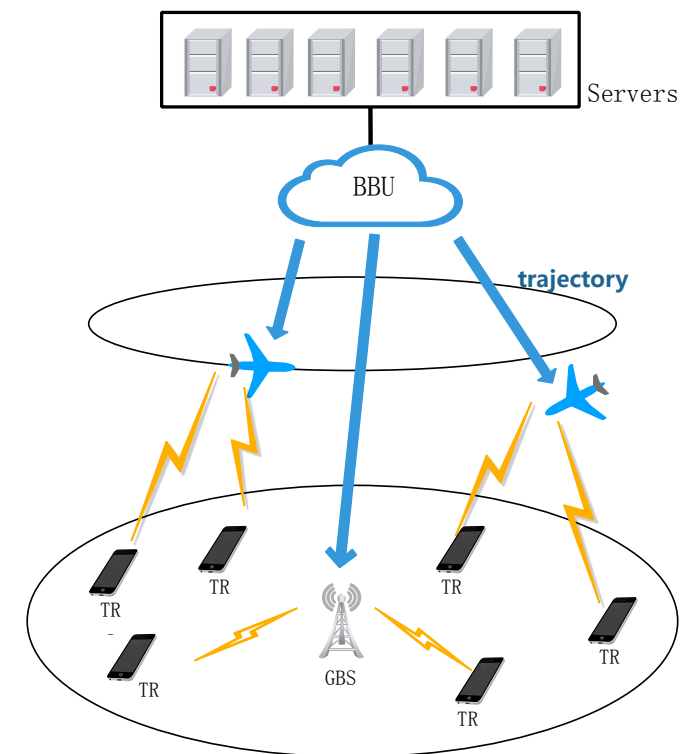

Figure 1. UAVs-enabled air-ground collaborative emergency network

\subsection{System Model}

Consider a set of UAVs $\mathcal{K}=\{1,2, \cdots, K\}$ and a ground base station serving a group of terminal receivers $\mathcal{M}=\{1,2, \cdots, M\}$ together. It is worth noting that $K \geq 1$ and $M \geq 1$. Without loss of generality, we assume that the GBS is located at the centre of the area, and the coordination is given by $c=\left[x_{G}, y_{G}\right]^{\mathrm{T}} \in \mathbb{R}^{2 \times 1}$. Suppose that the locations of terminal receivers can be obtained through some sort of positioning techniques and are represented as $w_{m}=\left[x_{m}, y_{m}\right]^{\mathbf{T}} \in \mathbb{R}^{2 \times 1}, \forall m \in \mathcal{M}$. UAVs are assumed to fly in a fixed horizontal plane with height $H$. Thus, the coordinate of UAV $k$ within the $n^{\text {th }}$ time slot is denoted by $q_{k}[n]=\left[x_{k}[n], y_{k}[n]\right]^{\mathrm{T}} \in \mathbb{R}^{2 \times 1}, \forall k \in \mathcal{K}, n \in \mathcal{N}$.

We study the data transmission over a period $T \geq 0$, during which all base stations (either aerial or terrestrial) disseminate information to TRs in an OFDMA manner. This saves us from considering the interference among different base stations. The selection of the value of $T$ needs to be cautious because it can have a great impact on the performance of the integrated network. A larger $T$ allows UAVs to get closer to TRs so as to achieve better channel quality. However, a larger $T$ also leads to larger latency. For ease of exposition, the period $T$ is divided into $N$ equal-length time slots, indexed by $n \in \mathcal{N}=\{1,2, \cdots, N\}$. The interval of each slot $\delta_{t}=T / N$ is set to be sufficiently small to make sure that UAVs can be seen as approximately stationary during each time slot even while flying at the top speed $V_{\max }[12]$. We define $\rho$ as the 
maximum ratio of the maximum travelling distance in a signal slot to the height of UAV. Thus, we have

$$
\frac{V_{\max } T}{N h} \leq \rho
$$

Then, we can get the minimum value as

$$
N=\operatorname{ceil}\left(\frac{V_{\max } T}{\rho h}\right)
$$

Where $\operatorname{ceil}(x)$ means taking the smallest integer no less than $x$.

The flight of UAVs must meet the actual physical constraints, which can be expressed as

$$
\begin{gathered}
S_{\min } \leq\left\|q_{k}[n+1]-q_{k}[n]\right\|_{2} \leq S_{\max }, \forall k, n=1,2, \cdots, N-1 \\
\left\|q_{k}[n]-q_{j}[n]\right\|_{2} \geq d_{\text {safe }}, \forall n, k \neq j
\end{gathered}
$$

Where $S_{\max } \triangleq V_{\max } \delta_{t}$ denotes the horizontal distance that UAVs can travel within a single time slot at top speed and $S_{\min } \triangleq V_{\min } \delta_{t}$. To avoid collision, the distance among two UAVs at any slot must be no less than the safe distance $d_{\text {safe }}$.

The Euclidean distance between UAV $k$ and TR $m$ at the $n^{\text {th }}$ time slot can be calculated as

$$
d_{k, m}[n]=\sqrt{H^{2}+\left\|q_{k}[n]-w_{m}\right\|_{2}^{2}}
$$

The mobility and height of UAV can guarantee high probability of creating LoS links. For simplicity, we assume UAVs and TRs always transmit data through LoS links, which means that the channel quality only relies on the UAV-TR distance. OFDMA can effectively eliminate the effects of multipath interference by inserting guard interval (GI). In addition, the Doppler effect caused by the fast movement of UAV can be handled well by some special techniques. Thus, the power gain of the channel between UAV $k$ to TR $m$ during time slot $n$ follows the free-space path loss model, which can be simplified as

$$
h_{k, m}[n]=\alpha_{0} d_{k, m}^{-2}[n]=\frac{\alpha_{0}}{H^{2}+\left\|q_{k}[n]-w_{m}\right\|_{2}^{2}}
$$

Where $\alpha_{0}$ is the channel power gain under the reference distance $d_{r}=1 \mathrm{~m}$.

As for the communication links between GBS and TRs, we can obtain the channel gain in a similar way, namely

$$
h_{g, m}[n]=\beta_{0} \eta\left\|w_{m}-c\right\|_{2}^{-n_{\beta}}
$$

Where the subscription $g$ represents the terrestrial base station. $\beta_{0}$ denotes the channel power gain under the reference distance $d_{r}=1 \mathrm{~m}$, and $n_{\beta}$ is the path loss exponent. $\eta$ in Equation (7) is introduced to imitate practical cases where there may be obstacles between GBS and TRs [13]. Normally, we set $\eta$ equal to 1 when there are not any obstacles, and we set $\eta$ less than 1 otherwise. The corresponding expression is

$$
\begin{cases}\eta=\eta_{0}, & \text { blocked } \\ \eta=1, & \text { otherwise }\end{cases}
$$

As mentioned previously, an OFDMA manner is adapted in this network. Assume that the maximum bandwidth a 
single base station can use is $B_{\max }$. To achieve synergy between UAVs and GBS, we introduce a set of bandwidth assignment variables $a_{k, m}[n], k \in \mathcal{K} \cup g, m \in \mathcal{M}, n \in \mathcal{N}$ to represent the proportion of bandwidth that each base station allocates to each link during the $n^{\text {th }}$ slot. The following principles are given: during each time slot, 1) every single TR can only communicate with a single base station, 2) every single aerial base station can only serve a single TR, and 3) the terrestrial base station can serve multiple TRs. These principles can be expressed as the following constraints:

$$
\left\{\begin{array}{l}
\sum_{k \in \mathcal{K} \cup g} a_{k, m}[n] \leq 1, \quad \forall m \in \mathcal{M}, n \in \mathcal{N} \\
\sum_{m \in \mathcal{M}} a_{k, m}[n] \leq 1, \forall k \in \mathcal{K} \cup g, n \in \mathcal{N} \\
a_{k, m}[n] \in\{0,1\}, \forall k \in \mathcal{K}, m \in \mathcal{M}, n \in \mathcal{N} \\
a_{g, m}[n] \in[0,1], \forall m \in \mathcal{M}, n \in \mathcal{N}
\end{array}\right.
$$

Here, $\mathcal{K} \cup g$ is the set of all kinds of base stations.

Define the downlink transmission power of UAV $k$ at the $n^{\text {th }}$ time slot as $p_{u, k}[n]$, which satisfies the constraint $0 \leq p_{u, k}[n] \leq p_{\max }$, as $p_{\max }$ signifies the maximum transmission power of UAVs. Consider the data dissemination between UAV $k$ and TR $m$ at the $n^{\text {th }}$ time slot. Because of the use of orthogonal carriers, we ignore the interference among different base stations. Therefore, the corresponding signal-to-noise ratio (SNR) at the receiver can be derived as

$$
\gamma_{k, m}[n]=\frac{p_{u, k}[n] h_{k, m}[n]}{\sigma^{2}}
$$

Where $\sigma^{2}$ signifies the variance of additive noise. Then, we can derive the corresponding available signal rate of TR $m$ as

$$
R_{k, m}[n]=B_{\max } a_{k, m}[n] \log _{2}\left(1+\gamma_{k, m}\right)
$$

Similarly, assuming that the transmission power of terrestrial base station is fixed as $p_{g}$, we can obtain the SNR and signal rate of the channel connecting the ground base station and TR $m$ as

$$
\begin{gathered}
\gamma_{g, m}[n]=\frac{p_{g}[n] h_{g, m}[n]}{\sigma^{2}} \\
R_{g, m}[n]=B_{\max } a_{g, m}[n] \log _{2}\left(1+\gamma_{g, m}\right)
\end{gathered}
$$

To meet every TR's communication demand, we hope to maximize the average signal rate of every TR in $\mathrm{N}$ slots. The average signal rate of TR $m$ can be calculated as follows:

$$
R_{m}=\frac{1}{N} \sum_{n \in \mathcal{N}}\left\{\sum_{k \in \mathcal{K}} R_{k, m}[n]+R_{g, m}[n]\right\}
$$

Because of the energy limit of UAVs, energy consumption for data dissemination of UAV $k$ in $N$ slots should be no larger than the given value $E_{\max }$.

$$
E_{k, t x}=\delta_{t} \sum_{n \in \mathcal{N}} p_{u, k}[n] \leq E_{\max }
$$

\subsection{Problem Formulation}

As discussed above, the goal of our work is to maximize the average signal rate of every TR by optimizing the bandwidth assignment, UAV trajectory, and transmission power. For ease of exposition, divide the variables into three groups, namely 
$A=\left\{a_{k, m}[n], a_{g, m}[n], \forall k, m, n\right\}$ and $Q=\left\{q_{k}[n], \forall k, n\right\}, P=\left\{p_{u, k}[n], \forall k, n\right\}$. Applying the formulas derived previously, we can obtain the following optimizing problem:

$\max _{\mathrm{A}, \mathrm{Q}, \mathrm{P}}$

$$
R_{m}=\frac{1}{N} \sum_{n \in \mathcal{N}}\left\{\sum_{k \in \mathcal{K}} R_{k, m}[n]+R_{g, m}[n]\right\}, \forall m \in \mathcal{M}
$$

s.t.

$$
\begin{gathered}
S_{\min } \leq q_{k}[n+1]-q_{k}[n] \|_{k} \leq S_{\max }, \forall k \in \mathcal{K}, n=1,2, \cdots, N-1 \\
\left\|q_{k}[n]-q_{j}[n]\right\|_{b} \geq d_{\text {safe }}, \quad \forall k, \mathrm{j} \in \mathcal{K}, k \neq j, n \in \mathcal{K} \\
\sum_{k \in \mathcal{K} \cup g} a_{k, m}[n] \leq 1, \forall m \in \mathcal{M}, n \in \mathcal{N} \\
\sum_{m \in \mathcal{M}} a_{k, m}[n] \leq 1, \forall k \in \mathcal{K} \cup g, n \in \mathcal{N} \\
a_{k, m}[n] \in\{0,1\}, \forall k \in \mathcal{K}, m \in \mathcal{M}, n \in \mathcal{N} \\
a_{g, m}[n] \in[0,1], \forall m \in \mathcal{M}, n \in \mathcal{N} \\
0 \leq p_{u, k}[n] \leq p_{\max }, \quad \forall k \in \mathcal{K}, n \in \mathcal{N} \\
E_{k, t x}=\delta_{t} \sum_{n \in \mathcal{N}} p_{u, k}[n] \leq E_{\max }, \forall k \in \mathcal{K}
\end{gathered}
$$

Objective functions are all non-convex, bandwidth assignment variables for UAVs are binary, and the others are continuous. Thus, this is a multi-objective mixed-integer non-convex optimization problem, which is difficult to solve directly to our best knowledge.

\section{Algorithm Design}

\subsection{Problem Pre-Processing}

To tackle the problem expressed in Equation (16), we first introduce an auxiliary variable $\varpi$ to turn the multi-objective problem into a single-objective problem by setting $\varpi$ to the maximum of all objectives. Secondly, the binary variables are relaxed in Equation (16e) into continuous variables ranging from 0 to 1 . Thus, Equation (16) can be reformulated as

$$
\text { A, }, \mathbb{Q}, \mathrm{x}, \varpi
$$

s.t.

$$
\begin{gathered}
R_{m}=\frac{1}{N} \sum_{n \in \mathcal{N}}\left\{\sum_{k \in \mathcal{K}} R_{k, m}[n]+R_{g, m}[n]\right\} \geq \varpi, \forall m \in \mathcal{M} \\
a_{k, m}[n] \in[0,1], \forall k \in \mathcal{K} \cap \mathrm{g}, m \in \mathcal{M}, n \in \mathcal{N} \\
\text { (16a), (16b), (16c), (16d), (16g), (16h) }
\end{gathered}
$$

Since the union of constraints (16e) and (16f) is a subset of constraint (17b), which is usually referred to as relaxation, we can easily infer that the solution to the problem expressed in Equation (17) serves as the upper bound of that expressed in Equation (16). The former is still non-convex due to constraints (17a) and (16b). Basically, there are no effective 
methods to solve these kinds of problems directly. However, there are some ingenious techniques that we can leverage to tackle this problem.

In the following, we propose an effective algorithm to deal with this optimization problem based on the block coordinate decent (BCD) method [14] and successive convex approximation (SCA). The core idea is to transform the original problem into three independent sub-problems equivalently. Specifically, we optimize three sets of variables alternatively. For instance, we fix the UAV trajectory and transmission power to optimize the bandwidth assignment. Then, we optimize the UAV trajectory under fixed transmission power and bandwidth assignment. The optimal solution obtained in the former sub-problem serves as the fixed variables in the latter sub-problem so as to achieve convergence along with iterations, which will be discussed later. It is worth noting that the sub-optimization problems may still be difficult to handle due to the non-convexity of objective function or constraints. Thus, successive convex approximation comes into use to eliminate non-convexity.

\subsection{Bandwidth Assignment Optimization}

With a given initial UAV trajectory and transmission power, i.e., $Q$ and $P$, Equation (17) is reduced to the bandwidth assignment optimization sub-problem as follows:

$\max _{A, \varpi} \varpi$

s.t.

$$
\begin{gathered}
R_{m}=\frac{B_{\max }}{N} \sum_{n \in \mathcal{N}}\left\{\sum_{k \in \mathcal{K}} a_{k, m}[n] \log _{2}\left(1+\gamma_{k, m}[n]\right)+a_{g, m}[n] \log _{2}\left(1+\gamma_{g, m}[n]\right)\right\} \geq \varpi, \forall m \in \mathcal{M} \\
a_{k, m}[n] \in[0,1], \forall k \in \mathcal{K} \cap g, m \in \mathcal{M}, n \in \mathcal{N} \\
\sum_{k \in \mathcal{K} \cup g} a_{k, m}[n] \leq 1, \forall m \in \mathcal{M}, n \in \mathcal{N} \\
\sum_{m \in \mathcal{M}} a_{k, m}[n] \leq 1, \forall k \in \mathcal{K} \bigcup g, n \in \mathcal{N}
\end{gathered}
$$

The problem expressed in Equation (18) is a standard linear programming (LP), since $a_{g, m}[n]$ can be seen as $a_{k, m}[n], k=g$. It can be solved directly by common optimization tools such as MATLAB optimization toolbox or CVX [12]. The optimal result of this bandwidth assignment optimization sub-problem is defined as $A^{*}$.

\subsection{UAV Trajectory Optimization}

With a given initial bandwidth assignment and transmission power, i.e., $A$ and $P$, Equation (17) is reduced to the UAV trajectory optimization sub-problem as follows:

$$
\text { Q, }
$$

s.t.

$$
\begin{gathered}
R_{m}=\frac{B_{\max }}{N} \sum_{n \in \mathcal{N}}\left\{\sum_{k \in \mathcal{K}} a_{k, m}[n] \log _{2}\left(1+\gamma_{k, m}[n]\right)+a_{g, m}[n] \log _{2}\left(1+\gamma_{g, m}[n]\right)\right\} \geq \varpi, \forall m \in \mathcal{M} \\
S_{\min } \leq\left\|q_{k}[n+1]-q_{k}[n]\right\|_{2} \leq S_{\max }, \forall k \in \mathcal{K}, n=1,2, \cdots, N-1 \\
\left\|q_{k}[n]-q_{j}[n]\right\|_{k} \geq d_{\text {safe }}, \forall k, \mathrm{j} \in \mathcal{K}, k \neq j, n \in \mathcal{K}
\end{gathered}
$$

The feasible solution sets that constraints (19a) and (19b) define are both non-convex, which define the non-convex 
optimization problem expressed in Equation (19). It is tough for non-convex optimization to obtain the exact global optimal solution. We can try to find a concave function that is as close as possible to the original function at a certain point. To seek out such a function, we consider a Taylor expansion.

Define

$$
r_{m, k}[n]=\log _{2}\left(1+\frac{p_{u, k}[n] \alpha_{0}}{\sigma^{2}\left(H^{2}+\left\|q_{k}[n]-w_{m}\right\|_{2}^{2}\right)}\right), \forall k, m, n
$$

Then, $R_{m}$ can be written as one constant plus the weighted sum of $N \times K$ parts with the same structure, i.e.,

$$
R_{m}=\sum_{n \in \mathcal{N}} \sum_{k \in \mathcal{K}} u_{m, k, n} r_{m, k}[n]+v_{m}
$$

Where $u_{m, k, n}=\frac{B_{\max }}{N} a_{k, m}[n]$ and $u_{m, k, n}=\frac{B_{\max }}{N} a_{k, m}[n], \forall m \in \mathcal{M}$. Because the nonnegative weighted sum of convex functions is still convex [15], we can just work on $r_{m, k}[n]$.

Observe that although $r_{m, k}[n]$ is not convex or concave with respect to $q_{k}[n]$, it is convex with respect to $\left\|q_{k}[n]-w_{m}\right\|_{2}^{2}$. The first-order Taylor expansion of any convex function is its own global lower-bound [15]. Therefore, if the point $q[n]_{k}^{i}$ in the $i^{\text {th }}$ iteration is known, we can obtain the lower bound for $r_{m, k}$ as follows:

$$
r_{m, k}^{i+1}[n] \geq r_{m, k}^{l b}[n]=\log _{2}\left(1+\frac{\varphi}{b+x^{i}}\right)-\frac{\varphi\left(x-x^{i}\right)}{\left(b+x^{i}\right)\left(b+x^{i}+\varphi\right) \ln 2}
$$

Where $\varphi=\frac{p_{u, k} \alpha_{0}}{\sigma^{2}}, b=H^{2}$, and $x=\left\|q_{k}[n]-w_{m}\right\|_{2}^{2}$. Since $\left\|q_{k}[n]-w_{m}\right\|_{2}^{2}$ is convex with respect to $q_{k}[n], r_{m, k}^{l b}[n]$ is concave with respect to $q_{k}[n]$.

Apply the first-order Taylor expansion at the given point $q_{k}^{i}[n], q_{j}^{i}[n]$ obtained at the $i^{\text {th }}$ iteration to $\left\|q_{k}[n]-q_{j}[n]\right\|_{2}^{2}$, we obtain [12]

$$
\left\|q_{k}[n]-q_{j}[n]\right\|_{2}^{2} \geq 2\left(q_{k}^{i}[n]-q_{j}^{i}[n]\right)^{T}\left(q_{k}[n]-q_{j}[n]\right)-\left\|q_{k}^{i}[n]-q_{j}^{i}[n]\right\|_{2}^{2}
$$

Then, Equation (19) can be reformulated as

$$
\text { Q, שax }
$$

s.t.

$$
\begin{gathered}
R_{m}^{l b}=\frac{B_{\max }}{N} \sum_{n \in \mathcal{N}}\left\{\sum_{k \in \mathcal{K}} a_{k, m}[n] r_{m, k}^{l b}+a_{g, m}[n] r_{g, m}\right\} \geq \varpi, \forall m \in \mathcal{M} \\
S_{\min } \leq\left\|q_{k}[n+1]-q_{k}[n]\right\|_{2} \leq S_{\max }, \forall k \in \mathcal{K}, n=1,2, \cdots, N-1 \\
2\left(q_{k}^{i}[n]-q_{j}^{i}[n]\right)^{T}\left(q_{k}[n]-q_{j}[n]\right)-\left\|q_{k}^{i}[n]-q_{j}^{i}[n]\right\|_{2}^{2} \geq d_{\text {safe }}, \forall k, \mathrm{j} \in \mathcal{K}, k \neq j, n \in \mathcal{K}
\end{gathered}
$$

Since the left hand of (24a) is affine and the left hand of (24c) is concave, the feasible solution sets defined here are both convex. Until now, this has been a more tractable problem. The original functions in constraints are replaced approximatively by their global under-estimators. As a consequence, the objective result of Equation (24) acts as the lower 
bound of that of Equation (19). The optimal result of this trajectory optimization sub-problem is defined as $Q^{*}$.

\subsection{UAV Transmission Power Optimization}

With a given initial bandwidth assignment and UAVs' trajectory, i.e., A and Q, Equation (17) is reduced to a transmission optimization sub-problem as follows:

$\max$

$\varpi$

s.t.

$$
\begin{gathered}
R_{m}=\frac{B_{\max }}{N} \sum_{n \in \mathcal{N}}\left\{\sum_{k \in \mathcal{K}} a_{k, m}[n] \log _{2}\left(1+\gamma_{k, m}[n]\right)+a_{g, m}[n] \log _{2}\left(1+\gamma_{g, m}[n]\right)\right\} \geq \varpi, \forall m \in \mathcal{M} \\
0 \leq p_{u, k}[n] \leq p_{\max }, \forall k \in \mathcal{K}, n \in \mathcal{N} \\
E_{k, t x}=\delta_{t} \sum_{n \in \mathcal{N}} p_{u, k}[n] \leq E_{\max }, \forall k \in \mathcal{K}
\end{gathered}
$$

Similarly, $R_{m}$ can be written as a form of weighted sum of several logarithmic functions. Let

$$
r_{m, k}^{\prime}[n]=\log _{2}\left(1+\frac{p_{u, k}[n] h_{k, m}[n]}{\sigma^{2}}\right), \forall m, k, n
$$

Then,

$$
R_{m}=\sum_{n \in \mathcal{N}} \sum_{k \in \mathcal{K}} u_{m, k, n}^{\prime} r_{m, k}^{\prime}[n]+v_{m}^{\prime}
$$

Where $u_{m, k, n}^{\prime}=\frac{B_{\max }}{N} a_{k, m}[n]$ and $v_{m}^{\prime}=\frac{B_{\max }}{N} \sum_{n \in \mathcal{N}} a_{g, m}[n] \log _{2}\left(1+\gamma_{g, m}[n]\right)$ are both constants. It is easy to prove that $r_{m, k}^{\prime}[n]$ is concave; thus, $R_{m}$ is concave. Then, the feasible solution set that $(25 \mathrm{a})$ defines is convex and makes the problem expressed in Equation (25) a convex optimization problem. The optimal result of this transmission power optimization subproblem is defined as $P^{*}$.

\subsection{Proposed Algorithm and Convergence Analysis}

On the basis of the derivations above, we propose an iterative update algorithm for Equation (25). The main idea is to transform the mixed-integer non-convex problem into three smooth convex optimization problems using the block coordinate decent method as well as convex successive convex approximation. To be specific, we solve the problems expressed in Equations (18), (24), and (25) alternatively to obtain the optimal solution of Equation (17). The detail of this algorithm is represented in Table 1.

Table 1. Iterative update algorithm

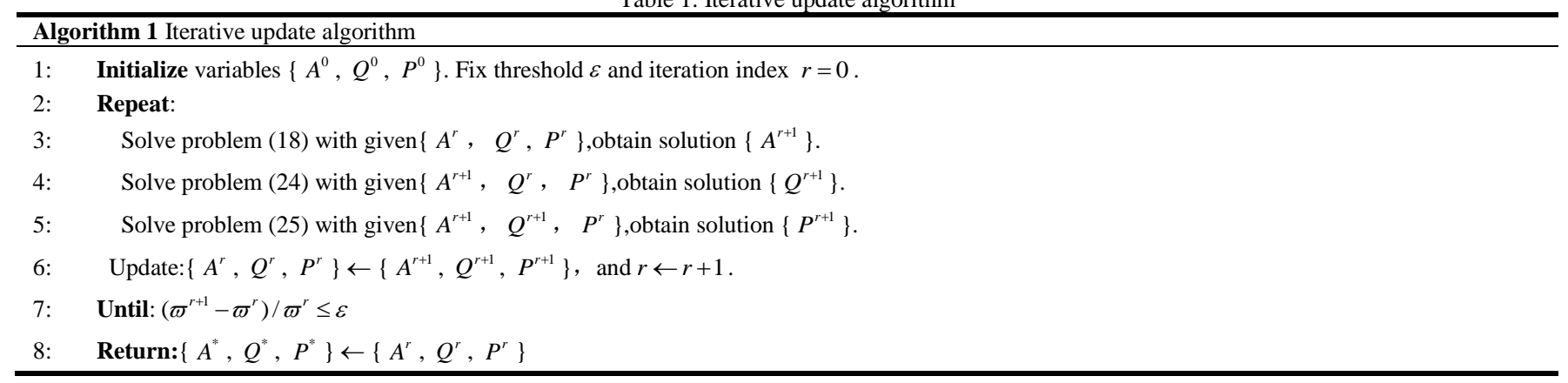


In classical block coordinate decent, if all the sub-problems are convex, then the convergence is guaranteed [16]. However, in Algorithm 1, we apply successive convex approximation when dealing with the trajectory optimization problem. Thus, we need to perform new convergence analysis on Algorithm 1. For the sake of description, we define the objective values under $A, Q, P$ of Equations (17), (18), (24), and (25) respectively as $\varpi(A, Q, P), \varpi_{\text {band }}(A, Q, P)$, $\varpi_{\text {tra }}(A, Q, P)$, and $\varpi_{\text {pow }}(A, Q, P)$. At first, there is no approximation, which means that the value of the objective function in Equation (18) is equal to that in Equation (17) under the same optimization variable value. Thus, we have

$$
\varpi\left(A^{r}, Q^{r}, P^{r}\right)=\varpi_{\text {band }}\left(A^{r}, Q^{r}, P^{r}\right)
$$

Secondly, the bandwidth assignment optimization problem is solved in step 4; thus, there exists

$$
\begin{gathered}
\varpi_{\text {band }}\left(A^{r}, Q^{r}, P^{r}\right) \leq \varpi_{\text {band }}\left(A^{r+1}, Q^{r}, P^{r}\right) \\
=\varpi\left(A^{r+1}, Q^{r}, P^{r}\right)
\end{gathered}
$$

The UAVs' trajectory is optimized with given $A^{r+1}, P^{r}$ in step 4, which follows that

(a)

$$
\varpi\left(A^{r+1}, Q^{r}, P^{r}\right)=\varpi_{t r a}\left(A^{r+1}, Q^{r}, P^{r}\right)
$$

(b)

$$
\leq \varpi_{\text {tra }}\left(A^{r+1}, Q^{r+1}, P^{r}\right)
$$

(c)

$$
\leq \varpi\left(A^{r+1}, Q^{r+1}, P^{r}\right)
$$

Where, in Equation (30), (a) holds because the first-order Taylor expansions applied in (22) and (23) are rigorous in the neighbourhood of given points respectively, which means that Equation (24) and Equation (19) have the same optimal objective value; (b) holds for the reason that the optimization of trajectory is effective; and (c) holds because of the fact that the objective result of Equation (24) acts as the lower bound of that of Equation (19), which was previously discussed. The inequality in (30) demonstrates that the objective value of the trajectory optimizing problem is still non-decreasing even though first-order Taylor expansion is adopted for approximation.

Finally, the optimization of transmission power is functional in step 5; thus, there exists

$$
\begin{gathered}
\varpi\left(A^{r+1}, Q^{r+1}, P^{r}\right)=\varpi_{\text {pow }}\left(A^{r+1}, Q^{r+1}, P^{r}\right) \\
\leq \varpi_{\text {pow }}\left(A^{r+1}, Q^{r+1}, P^{r+1}\right) \\
\leq \varpi\left(A^{r+1}, Q^{r+1}, P^{r+1}\right)
\end{gathered}
$$

Based on the above discussion, we have

$$
\varpi\left(A^{r}, Q^{r}, P^{r}\right) \leq \varpi\left(A^{r+1}, Q^{r+1}, P^{r+1}\right)
$$

Equation (32) suggests that the optimal objective value of Equation (17) in each iteration is non-decreasing. In addition, because of the limited resources, it is evident that the objective function of Equation (17) is upper bounded. Therefore, the algorithm proposed is bound to converge.

\section{Simulation Results}

Consider a scenario with $M=6$ terminal receivers that are randomly distributed within a square area of $2 \mathrm{~km} \times 2 \mathrm{~km}$ on the ground. All the UAVs are assumed to fly in a horizontal plane at an altitude $H=100 \mathrm{~m}$. The channel power gain of UAVTR link and GBS-TR link under the reference distance $d_{0}=1 \mathrm{~m}$ are respectively set as $\alpha_{0}=-60 \mathrm{~dB}$ and $\beta_{0}=-40 \mathrm{~dB}$. The path loss exponent of the GBS-TR link and the receiver noise power are assumed as $n_{\beta}=3$ and $\sigma^{2}=-167 \mathrm{dBm}$, respectively. Assume that one TR (e.g., 2 or 4 ) suffers from severe channel attenuation $\eta_{b}=-10 \mathrm{~dB}$ because of the blockage 
of obstacles between GBS-TR links. The threshold $\varepsilon$ is set as $10^{-6}$. Other parameters are set as $0 \leq p_{k}[n] \leq 100 \mathrm{~mW}$, $p_{g}=300 \mathrm{~mW}, d_{\text {safe }}=80 \mathrm{~m}$, and $\rho=0.5$. Simulative experiments are carried out based on one possible distribution of the TRs locations, as shown in Figure 3.

In Figure 3, the optimal trajectories obtained by Algorithm 1 under different periods $\mathrm{T}$ are plotted in different colors. It is observed that as $\mathrm{T}$ increases, the UAV adjusts its trajectory to move closer to the terminal receivers so as to achieve a larger signal rate. When $\mathrm{T}$ is sufficiently large, the UAV is able to cover all the TRs and hover above each of them at the minimum speed to make the best of the best communication channels. This can be seen in Figure 4 . When traveling between the TR locations, UAVs fly at the maximum speed to become close to TRs faster and save time for better channels.

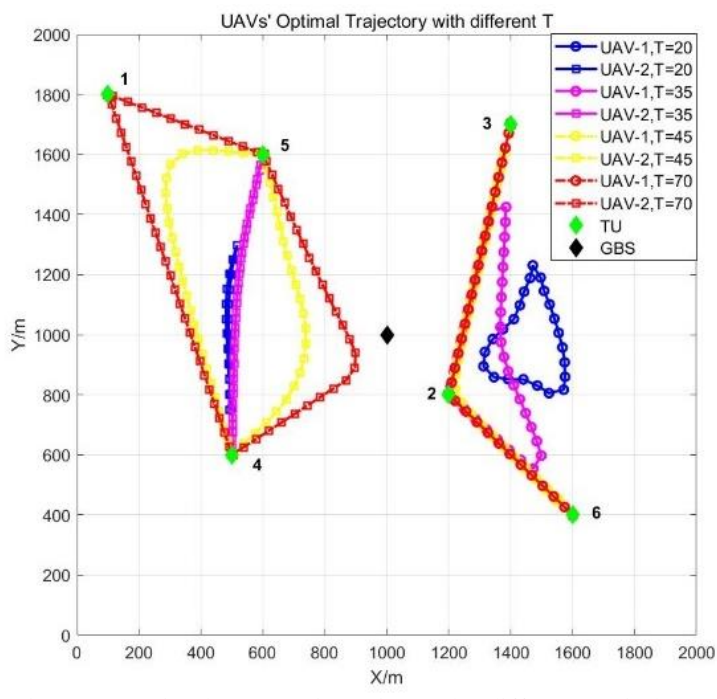

Figure 3. Optimal UAV trajectories under different periods T


Figure 4. The UAVs' speed versus time under $\mathrm{T}=60 \mathrm{~s}$

Figure 5 shows the optimal objective function value under different periods $T$. With an increase in $T$, the achievable signal rate of TR also increases, but the growth slows down gradually. This is because a large T allows UAVs to move closer to TRs and achieve a better quality of service. The signal rate begins to drop off after $T=75 \mathrm{~s}$. This is because the energy consumption of transmission is restricted, as UAVs are able to cover all the TRs, and continuing to increase the period $T$ will result in the decline of available energy in each slot. When the improvement of channel quality cannot make up for the influence caused by the decline in available energy, the average signal rate will decrease. The results suggest that we need to set $T$ according to the actual scenario, and a large $T$ is not always better.

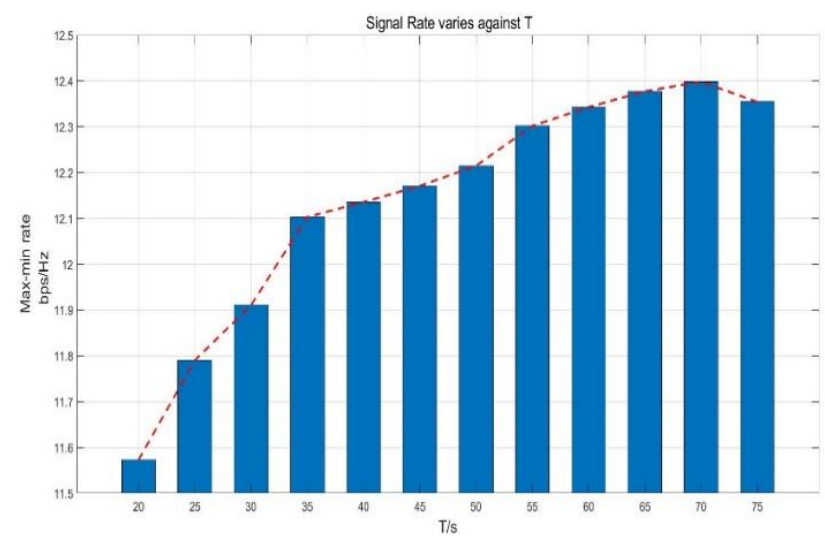

Figure 5. Optimal objective function value under different periods $\mathrm{T}$

Figure 6 exhibits the optimal bandwidth assignment obtained by Algorithm 1. The darker the fill color is in the cell, the more bandwidth that is assigned to the corresponding channel. The GBS does not serve TR 2 since the existence of severe blockage has a great effect on the quality of communication. Instead, UAV-1 is in charge of the data dissemination to TR 2. 
Since the GBS can communicate with multiple TRs simultaneously and has abundant energy, it makes use of every slot to transmit data to vacant TRs with the purpose of maximizing the average signal rate of each TR over period T. Figure 7 shows the transmission power in different slots. We can conclude from the diagram that energy consumption must reach a balance among different slots due to the energy budget. Figure 8 illustrates the great performance of convergence of the proposed Algorithm 1.
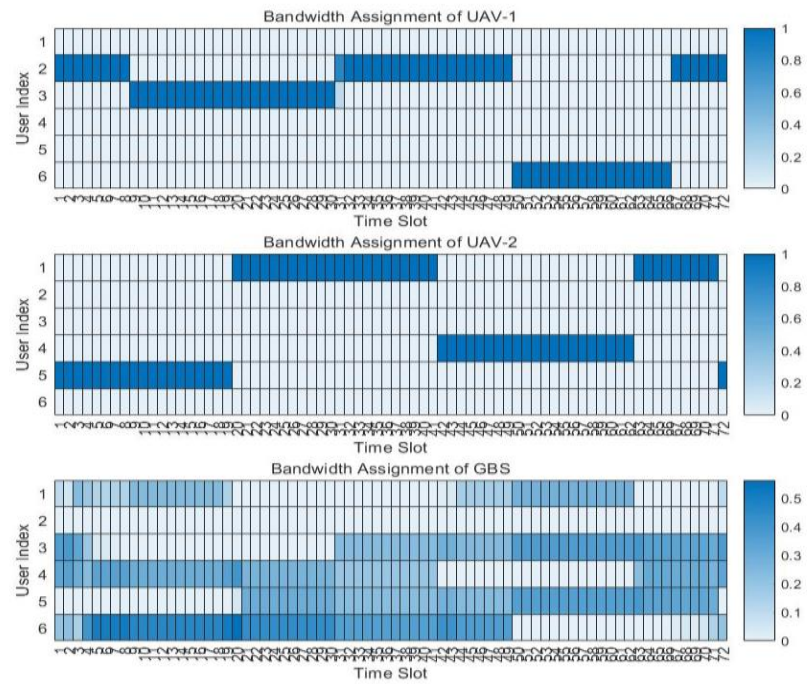

Figure 6. Optimal bandwidth assignment results under $T=60 \mathrm{~s}$
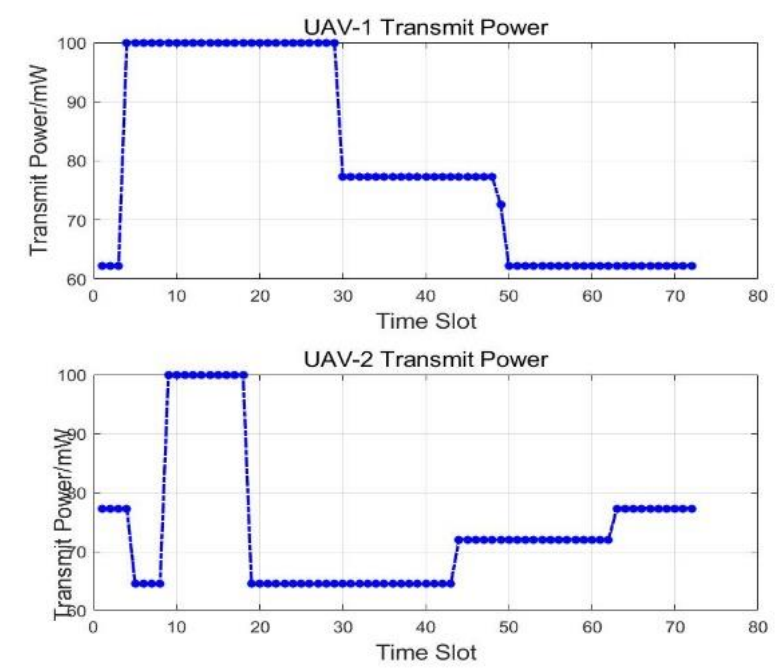

Figure 7. The UAVs' transmission power versus time under $T=60 \mathrm{~s}$

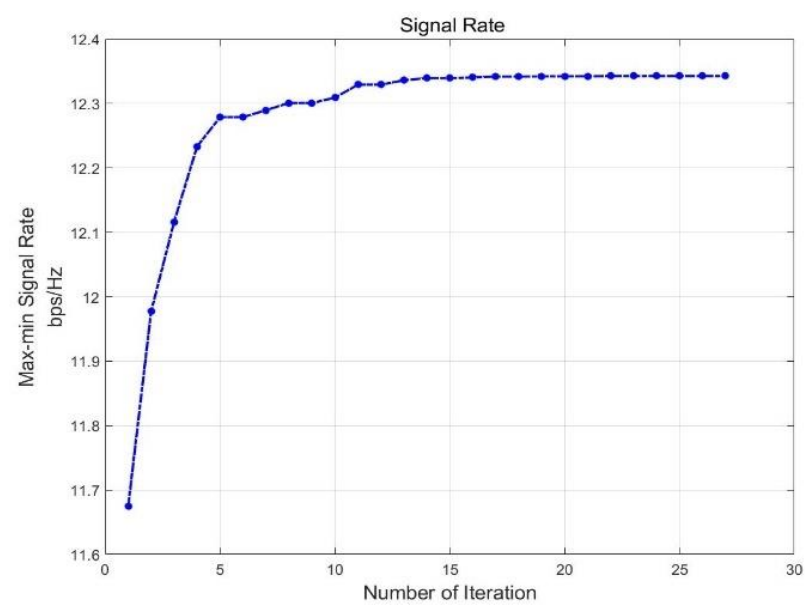

Figure 8. Convergence performance of the proposed algorithm

In Table 2, we design the following four schemes for a two-UAV network for comparison, namely 1) Scheme I: Optimize the UAVs' trajectory and transmission power with a given bandwidth assignment. 2) Scheme II: Optimize the bandwidth assignment as well as the transmission power with a fixed location of stationary UAVs. 3) Scheme III: Optimize the bandwidth assignment as well as the transmission power with a fixed trajectory of mobile UAVs. 4) Scheme IV: All variables are jointly optimized as shown in Algorithm 1. From Table 2, we can draw several important conclusions. Firstly, the comparison of Scheme III and Scheme I shows that the optimization of bandwidth assignment makes a great difference. Secondly, compared with that of either Scheme II or Scheme III, the signal rate of Scheme IV has significant improvement, which proves that the trajectory optimization is effective. Thirdly, the improvement of the signal rate of Scheme III relative to Scheme II is limited. There are two reasons accounting for this. First, we simplified the signal fading model in this paper and the scope of simulation scene is small, so the influence caused by the change in distance between UAV and TR is small. Second, the optimization performance of the bandwidth assignment is outstanding, which can alleviate the performance decline caused by a fixed location. To sum up, the proposed joint optimization scheme of bandwidth assignment, UAVs' trajectory, and transmission power can achieve significant improvements of network performance. 
Table 2. Comparison of optimal results from different schemes

\begin{tabular}{ccc}
\hline Scheme & Signal rate (bps/Hz) & Growth proportion \\
\hline Scheme I & 9.6328 & - \\
Scheme II & 11.7356 & $+0.88 \%$ (Compared with Scheme II) \\
Scheme III & 11.8393 & $+28.13 \%$ (Compared with Scheme I) \\
& & $+5.17 \%$ (Compared with Scheme II) \\
Scheme IV: & 12.3426 & $+4.25 \%$ (Compared with Scheme III) \\
& & with \\
\hline
\end{tabular}

\section{Conclusions}

This study sought to optimize resource allocation in an air-ground integrated network with multiple UAVs and one ground base station. An effective iterative updating algorithm was designed based on BCD and SCA to solve the formulated complex optimization problem. The simulation results illustrate that compared with the partial optimization strategy, the proposed algorithm can improve the average signal rate over a period $\mathrm{T}$ of all terminal receivers by $5 \%-28 \%$, and the proposed algorithm has great convergence performance. Furthermore, the results suggest that it is important to choose an appropriate value for $\mathrm{T}$ to fully exploit the mobility potential of UAVs. However, due to the lack of considering the limit of the UAV's acceleration, there was a sudden change in the UAV's speed, which needs to be fixed in future work.

\section{References}

1. Y. Zeng, R. Zhang, and T. J. Lim, "Wireless Communications with Unmanned Aerial Vehicles: Opportunities and Challenges," IEEE Communications Magazine, Vol. 54, No. 5, pp. 36-42, 2016

2. M. Mozaffari, W. Saad, M. Bennis, and M. Debbah, "Drone Small Cells in the Clouds: Design, Deployment and Performance Analysis," in Proceedings of 2015 IEEE Global Communications Conference, pp. 1-6, IEEE, 2015

3. C. T. Cicek, H. Gultekin, B. Tavli, and H. Yanikomeroglu, "UAV Base Station Location Optimization for Next Generation Wireless Networks: Overview and Future Research Directions," in Proceedings of the $1^{\text {st }}$ IEEE Unmanned Vehicle Systems (UVS) Conference, 2018

4. M. Mozaffari, W. Saad, M. Bennis, Y. H. Nam, and M. Debbah, "A Tutorial on UAVs for Wireless Networks: Applications, Challenges, and Open Problems," IEEE Communications Surveys \& Tutorials, Vol. 21, No. 3, pp. 2334-2360, 2019

5. A. Al-Hourani, S. Kandeepan, and S. Lardner, "Optimal LAP Altitude for Maximum Coverage," IEEE Wireless Communications Letters, Vol. 3, No. 6, pp. 569-572, 2014

6. M. Mozaffari, W. Saad, M. Bennis, and M. Debbah, "Efficient Deployment of Multiple Unmanned Aerial Vehicles for Optimal Wireless Coverage," IEEE Communications Letters, Vol. 20, No. 8, pp. 1647-1650, 2016

7. R. I. Bor-Yaliniz, A. El-Keyi, and H. Yanikomeroglu, "Efficient 3-D Placement of an Aerial Base Station in Next Generation Cellular Networks," in Proceedings of 2016 IEEE International Conference on Communications (ICC), pp. 1-5, IEEE, 2016

8. Y. Zeng, R. Zhang, and T. J. Lim, "Throughput Maximization for UAV-Enabled Mobile Relaying Systems," IEEE Transactions on Communications, Vol. 64, No. 12, pp. 4983-4996, 2016

9. Y. Zeng and R. Zhang, "Energy-Efficient UAV Communication with Trajectory Optimization," IEEE Transactions on Wireless Communications, Vol. 16, No. 6, pp. 3747-3760, 2017

10. J. Lyu, Y. Zeng, and R. Zhang, "Cyclical Multiple Access in UAV-Aided Communications: A Throughput-Delay Tradeoff," IEEE Wireless Communications Letters, Vol. 5, No. 6, pp. 600-603, 2016

11. Z. Xue, J. Wang, G. Ding, H. B. Zhou, and Q. H. Wu, "Maximization of Data Dissemination in UAV-Supported Internet of Things," IEEE Wireless Communications Letters, Vol. 8, No. 1, pp. 185-188, 2019

12. Q. Wu, Y. Zeng, and R. Zhang, "Joint Trajectory and Communication Design for Multi-UAV Enabled Wireless Networks," IEEE Transactions on Wireless Communications, Vol. 17, No. 3, pp. 2109-2121, 2018

13. Z. Xue, J. Wang, G. Ding, H. B. Zhou, and Q. H. Wu, "Cooperative Data Dissemination in Air-Ground Integrated Networks," IEEE Wireless Communications Letters, Vol. 8, No. 1, pp. 209-212, 2019

14. Y. Xu and W. Yin, "A Block Coordinate Descent Method for Regularized Multiconvex Optimization with Applications to Nonnegative Tensor Factorization and Completion," SIAM Journal on Imaging Sciences, Vol. 6, No. 3, pp. 1758-1789, 2013

15. S. Boyd and L. Vandenberghe, "Convex Optimization," Cambridge University Press, Cambridge, U.K, 2004

16. P. Jain and P. Kar, "Non-Convex Optimization for Machine Learning," Foundations and Trends® in Machine Learning, Vol. 10, No. 3-4, pp. 142-336, 2017 